\title{
La Industria 4.0 en el Mantenimiento Industrial
}

\section{Industry 4.0 in Industrial Maintenance}

MERAZ-MENDEZ, Manuel†*, LERMA-HERNANDEZ, Claudia y CORRAL-RAMIREZ, Guadalupe

Universidad Tecnológica de Chihuahua, Avenida Montes Americanos 9501, Sector 35, C.P.31216

ID $1^{\text {er }}$ Autor: Manuel, Meraz-Mendez / ORC ID: 0000-0001-8254-957, Researcher ID Thomson: S-4565-2018, CVU CONACYT ID: 250582

ID $1{ }^{\text {er }}$ Coautor: Claudia, Lerma-Hernández / ORC ID: 0000-0003-3232-0431, CVU CONACYT ID: 216319

ID $2^{\text {do }}$ Coautor: Guadalupe, Corral-Ramírez / ORC ID: 0000-0003-4874-4036, CVU CONACYT ID: 520946

DOI: $10.35429 /$ JIE.2019.7.3.17.24

Recibido 15 de Enero, 2019; Aceptado 22 Marzo, 2019

\section{Resumen}

La industrial 4.0 es la incorporación de tecnologías digitales en las fábricas como: la inteligencia artificial, machine learning, impresión 3D, drones, robótica, IOT, big data, realidad virtual, automatización, entre otros, que tienen como objetivo la digitalización de los procesos productivos en las fábricas, a estas también se les denomina fábricas inteligentes (Garatu, 2016). El presente artículo tiene como objetivo identificar las tecnologías aplicables al mantenimiento industrial en la industria 4.0, para determinar si los docentes de la Carrera de Ingeniería en Mantenimiento Industrial de la Universidad Tecnológica de Chihuahua están aplicando en sus prácticas de enseñanza estas tecnologías usadas por empresas que utilizan Industria 4.0, como resultado final de esta investigación se identificaran los factores que involucran el por qué no se aplican estas tecnologías en el aula, así como las competencias y habilidades que necesitan los estudiantes para enfrentar este reto, para finalmente dar una recomendación a la institución para lograr el objetivo a cumplir con las necesidades de la industria de la región.

Industria 4.0, Mantenimiento, Nuevas prácticas

\begin{abstract}
Industry 4.0 is the incorporation of digital technologies in factories such as: artificial intelligence, machine learning, 3D printing, drones, robotics, IOT, big data, virtual reality, automation, among others, which aim to digitalize processes productive in the factories, these are also called smart factories. The objective of this article is to identify the technologies applicable to industrial maintenance in Industry 4.0, the final result of this research determine the teaching practices that must be carried out in the Industrial Maintenance Engineering career at the Technological University of Chihuahua, and how the students must be prepared with the competences and skills necessary to face this challenge, at the same time the new teaching practices and strategies that a teacher in the technical area of Industrial Maintenance must apply in laboratory practices with a focus on Industry 4.0.
\end{abstract}

Industry 4.0, Maintenance, Teaching practices

Citación: MERAZ-MENDEZ, Manuel, LERMA-HERNANDEZ, Claudia y CORRAL-RAMIREZ, Guadalupe. La Industria 4.0 en el Mantenimiento Industrial. Revista de Ingeniería Industrial. 2019. 3-7: 17-24

\footnotetext{
*Correspondencia al Autor (Correo Electrónico: mmeraz@utch.edu.mx)

$\dagger$ Investigador contribuyendo como primer autor.
} 


\section{Introducción}

Hoy en día, es muy común hablar de la Industria 4.0 (I4.0), también llamada la cuarta revolución industrial, en la cual las grandes fábricas Industriales cuentan en sus procesos de producción la intercomunicación de equipos, máquinas y líneas de producción, esto ofrece la ventaja de una fábrica flexible para seguir adaptándose a las circunstancias y conseguir en todo momento el máximo rendimiento, las nuevas tecnologías se basan en la conexión a internet y la transferencia de datos se realiza de manera digital, esto quiere decir que no es necesario hacer intervenciones en la maquinaria para poder tomar mediciones de esos parámetros físicos, las máquinas ya se los comunican entre sí, y de esta manera lo aprovechamos para aplicar el mantenimiento industrial de I4.0 (Ibérica, 2018)

Por lo tanto, el tipo de mantenimiento industrial más adecuado para las fábricas inteligentes es precisamente el mantenimiento predictivo. Gracias a softwares de análisis, se tiene en todo momento los datos del estado de las máquinas, el sistema informático ayuda a predecir cuándo una máquina tiene desgaste o presenta una falla o anomalía en su funcionamiento. También ayuda a decidir cuándo es el momento idóneo para hacer una reparación o sustitución antes de que se produzca una avería que ponga en riesgo el buen funcionamiento de la fábrica., además, gracias a las particularidades de la I4.0, el resto de la maquinaria podrá adaptarse a la circunstancia y conseguir el máximo rendimiento mientras esa parte del proceso de producción está en reparación.

¿Pero están las instituciones educativas encargadas de formar profesionales técnicos e ingenieros preparados para formar a sus estudiantes ante este nuevo reto? En caso particular, la Universidad Tecnológica de Chihuahua (UTCH) sus programas de estudio se han actualizado con un enfoque en la I4.0, es por ellos que en esta investigación se identificara el papel del Mantenimiento en la I4.0,que permita identificar el grado de conocimiento de los maestros en esta nueva era y si están preparados técnicamente, para proponer que tipo de prácticas de enseñanza los docentes deben aplicar en sus materias para impartir la cátedra con un enfoque centrado en la I4.0.

\section{Objetivo}

Identificar las tecnologías aplicadas al mantenimiento en la industria 4.0 susceptibles de ser utilizadas por los docentes en las prácticas de enseñanza en la carrera de Ingeniería en Mantenimiento Industrial de la UTCH.

\section{Metodología}

La metodología que se utiliza en esta investigación, como se observa en la figura 1, consta de una serie de pasos y tiene un enfoque de tipo descriptivo mismo que permite identificar la utilización del mantenimiento en la industria 4.0 para que docentes y alumnos de la carrera de Mantenimiento Industrial de la UTCH lo apliquen en su quehacer académico.

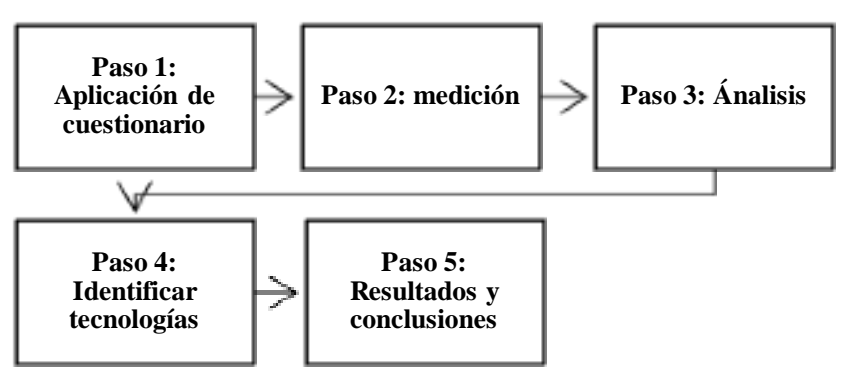

Figura 1 Metodología y pasos a seguir

\section{Paso 1 aplicación del cuestionario}

Para esta investigación el siguiente cuestionario (Tabla 1.) fue aplicado a docentes de la carrera de Mantenimiento Industrial de la UTCH con el objetivo de determinar el grado de conocimientos de la I4.0 y su aplicación en la práctica docente.

\begin{tabular}{|c|c|c|}
\hline \multicolumn{3}{|l|}{ Cuestionario $\mathbf{I 4 . 0}$} \\
\hline & $\mathrm{Si}$ & No \\
\hline 1. - ¿Conoce el término Industria 4.0? & 14 & 5 \\
\hline 2.- Conoce las tecnologías de la I4.0: & 9 & 10 \\
\hline $\begin{array}{l}\text { 3.- Ha aplicado algunas tecnologías } \\
\text { de la I4.0 a su práctica docente: }\end{array}$ & 4 & 15 \\
\hline $\begin{array}{l}\text { 4.- Ha recibido alguna capacitación } \\
\text { de tecnologías de la Industria } 4.0\end{array}$ & 4 & 15 \\
\hline $\begin{array}{l}\text { 5.- Cuenta la UTCH con la tecnología } \\
\text { I4.0 }\end{array}$ & 6 & 13 \\
\hline 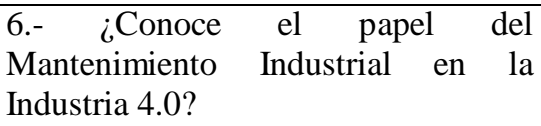 & 7 & 12 \\
\hline
\end{tabular}

Tabla 1 Cuestionario de la Industria 4.0 


\section{Paso 2. Mediciones}

Las siguientes graficas muestran el resultado de la aplicación del cuestionario a una muestra de 20 profesores de la carrera de Mantenimiento Industrial.

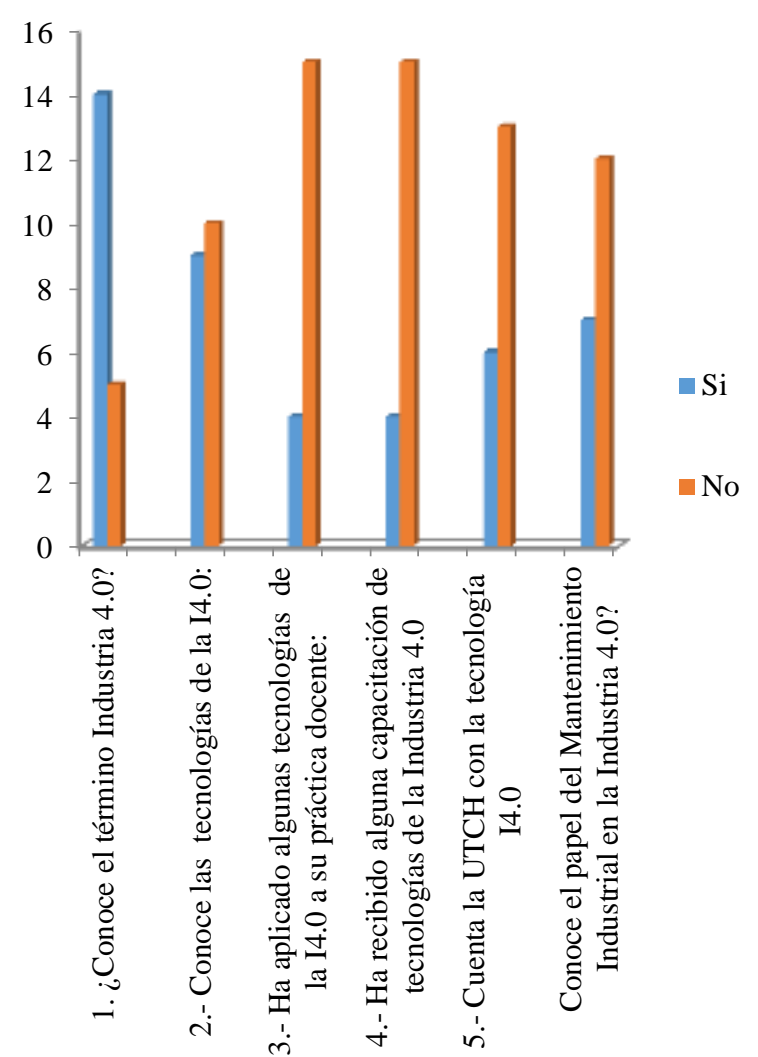

Gráfica 1 Tecnologías de la Industria 4.0 que más se conocen por los docentes

Fuente: Cuestionario Industria 4.0 en la educación

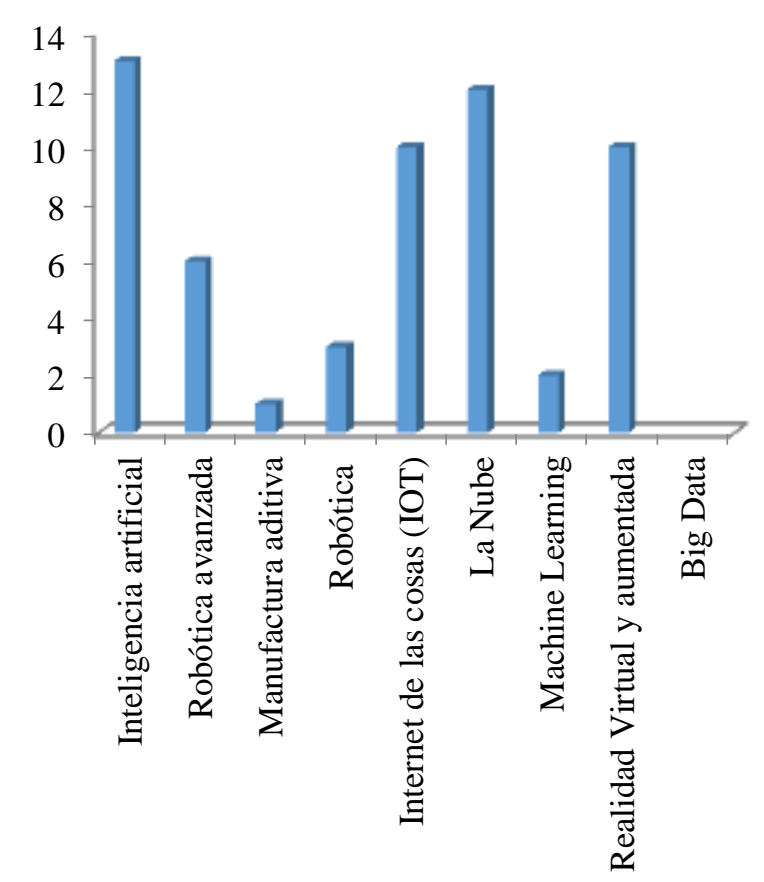

Gráfica 2 Tecnologías de la Industria 4.0 que más se conocen por los docentes

Fuente: Cuestionario Industria 4.0 en la educación

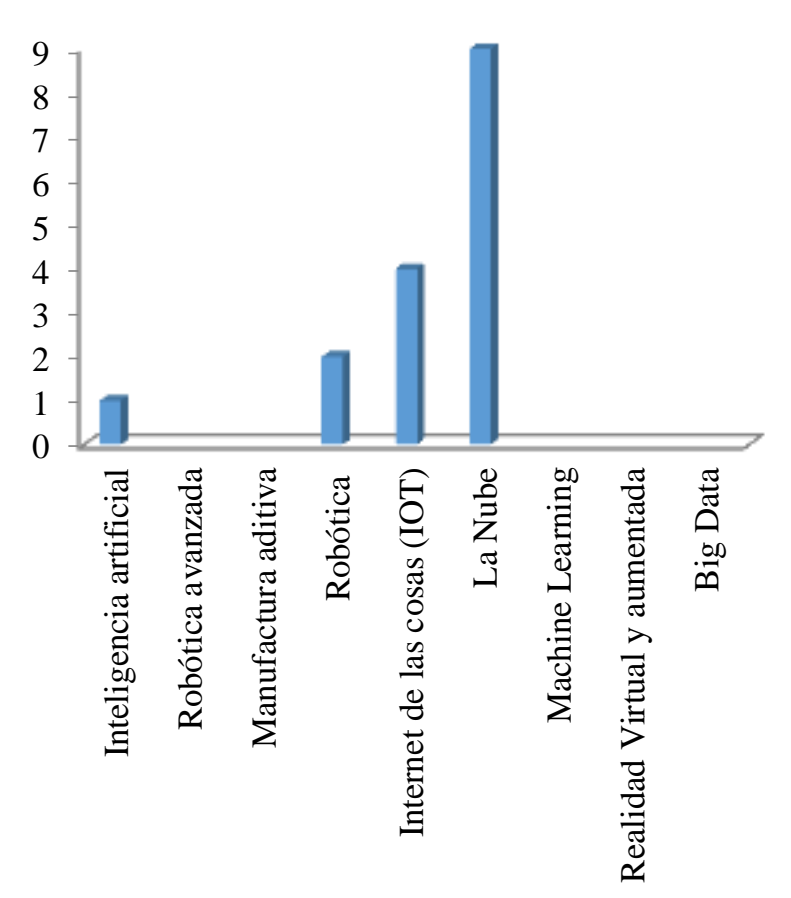

Gráfica 3 Tecnologías de la I4.0 que aplican los maestros en su práctica docente

Fuente: Cuestionario Industria 4.0 en la educación

\section{Paso 3 Análisis}

La gráfica 2 muestra las tecnologías de la Industria 4.0 que más se conocen por los docentes entrevistados en la carrera de Mantenimiento Industrial, en ella se muestra que la mayoría conoce que es la industria 4.0 por que han oído hablar en las noticias o en algunas conferencias que han ido en los últimos 3 años, pero aun así no han recibido capacitación alguna por parte de la universidad y por consecuente no las están aplicando en su práctica docente, aunado a ello más del $50 \%$ de los docentes no tienen idea de cómo se aplica el mantenimiento en la industria 4.0

La grafica 3 muestra las tecnologías que más conocen los docentes de la carrera de Mantenimiento Industrial, en ella se observa que las tecnologías que más conocen son la Inteligencia artificia, la nube, el internet de las cosas (IOT) y la realidad virtual, ya que estas la aplican más en su vida diaria como por ejemplo subir fotos y archivos a la nube (Dropbox, Microsoft One Drive, Google Drive, etc.), videojuegos, compras en línea (mercado libre, Amazon, Ebay, etc.), pedir un viaje (Uber y Didi) y hasta el pago de servicios por internet (Luz, agua, teléfono, Liverpool, etc.).

MERAZ-MENDEZ, Manuel, LERMA-HERNANDEZ, Claudia y CORRAL-RAMIREZ, Guadalupe. La Industria 4.0 en el Mantenimiento Industrial. Revista de Ingeniería Industrial. 2019 
La grafica 4 muestra las tecnologías que aplican los maestros en su práctica docente, en la cual se puede observar que la nube es la que más aplican ya que algunos docentes entrevistados dicen que la aplican para que enviar y recibir tareas y trabajos de los alumnos. Muy pocos docentes han aplicado IOT, Inteligencia artificial y Robótica.

\section{Resultado:}

Las gráficas anteriores nos dan como resultado la poca aplicación que los docentes hacen de estas tecnologías y demuestra que no estamos aún preparados para formar a nuestros estudiantes con conocimientos a esta nueva era de I4.0.

El resultado final es que los docentes no tienen el conocimiento y la capacitación de las tecnologías que utilizan las industrias que utilizan el I4.0 en el Mantenimiento Industrial, es por ello que es necesario identificar estas tecnologías y aplicarlas a nuestra práctica docente en la medida que vayamos avanzando.

\section{Paso 4. Identificar las tecnologías de la Industria 4.0 aplicadas al Mantenimiento Industrial}

\section{Software de sistema de gestión de mantenimiento inteligente}

Están diseñados principalmente para un mantenimiento preventivo y dependiente del rendimiento con el objetivo de maximizar la disponibilidad de la planta (Siemens, 2019), en la figura 2 se muestra el sistema y sus aplicaciones.

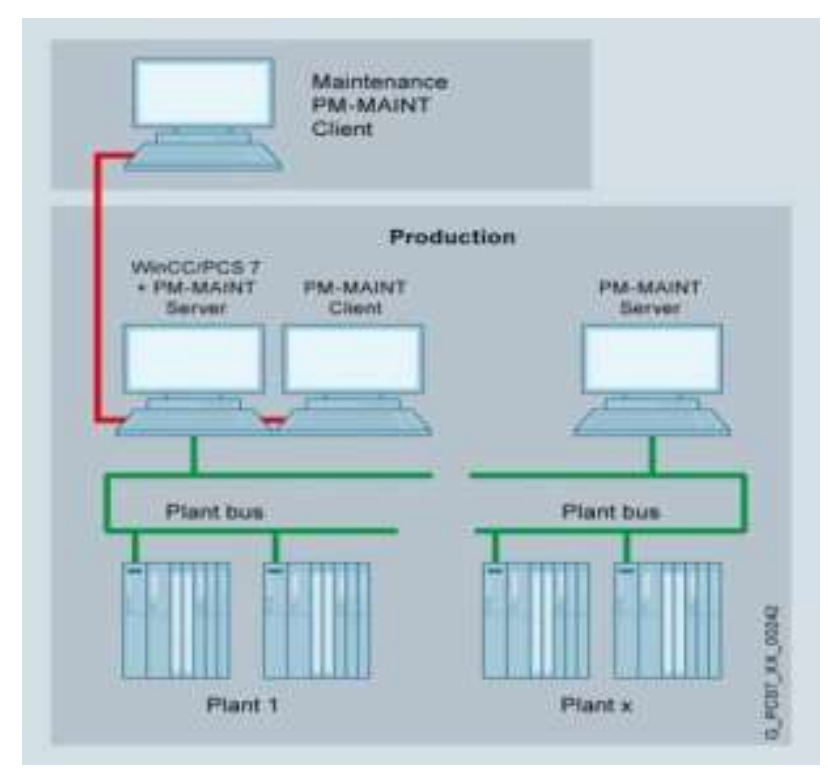

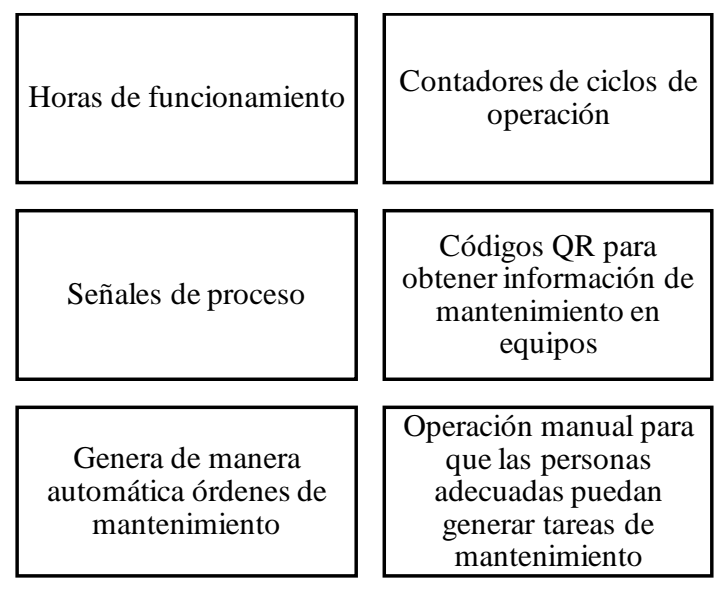

Figura 2 Software PM MAINT y Aplicaciones Fuente: Cortesía Siemens Win CC

\section{Software para análisis estadístico}

Realiza un análisis estadístico de los parámetros de operación y alarmas de equipos en el proceso para tomar acciones al respecto (Siemens, PM-ANALIZAR, 2019), en la figura 3 se muestra el sistema y sus aplicaciones.
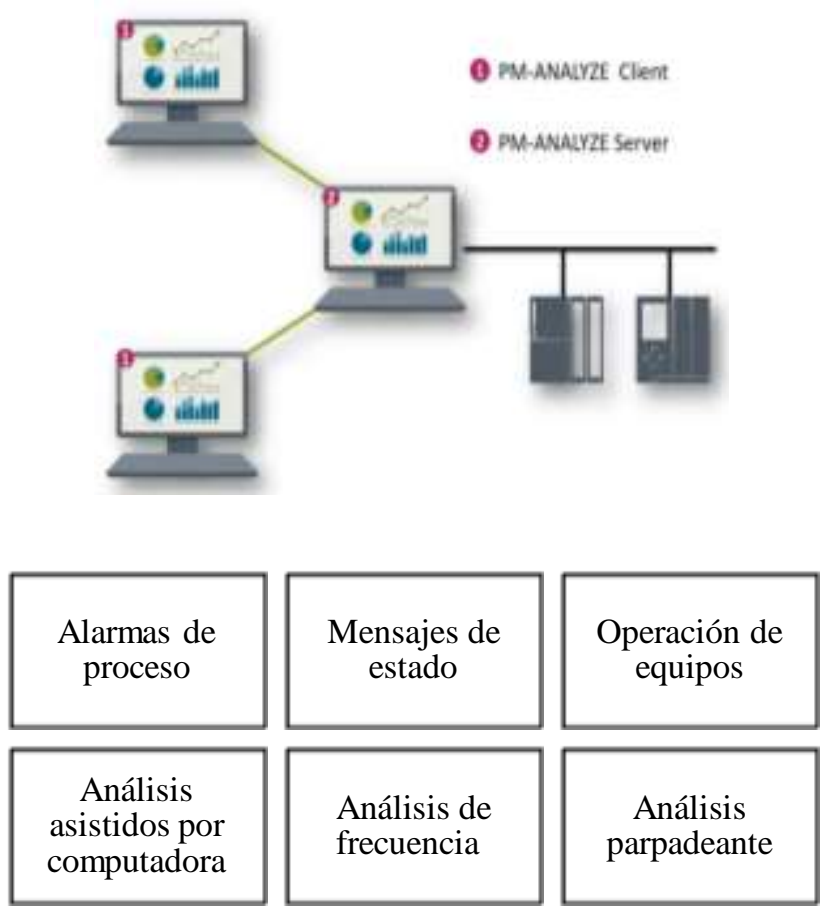

Figura 3 Software PM ANALYZE y Aplicaciones Fuente: Cortesía Siemens Win CC

\section{Panel HMI}

Permite monitorear y visualizar errores en el proceso directamente en el equipo y por medio de la conexión al PLC podemos visualizar en el programa donde ocurrió la falla, en la figura 4 se muestra un panel HMI y sus aplicaciones. 

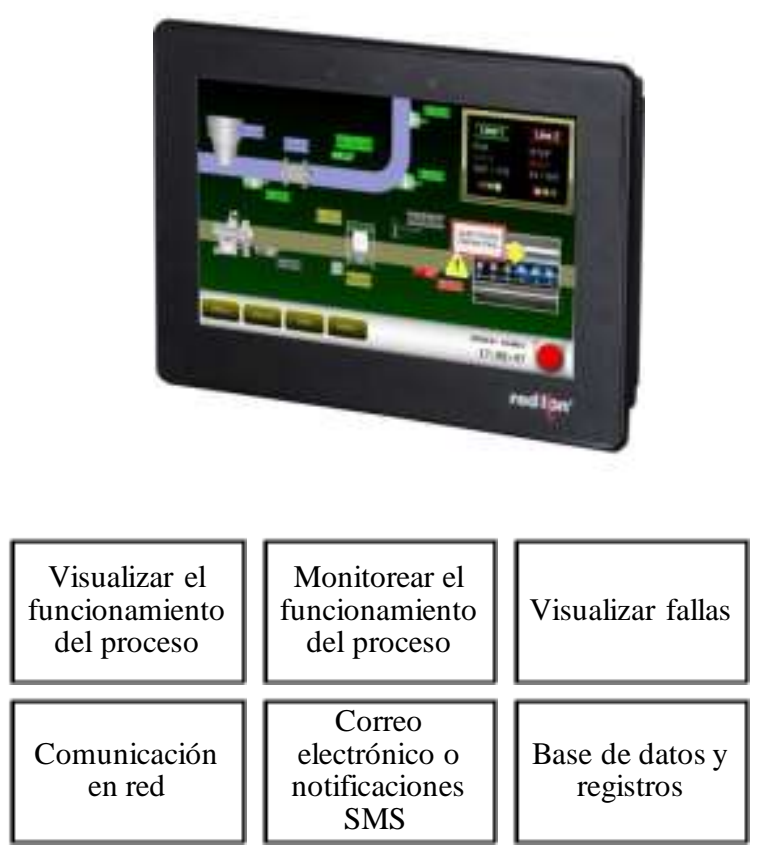

Figura 4 Panel HMI y Aplicaciones Fuente: Cortesía Redlion

\section{La nube}

Subir datos a la nube para que por medio de la inteligencia artificial se puedan tomar decisiones, en la figura 5 se muestra el sistema y sus aplicaciones.
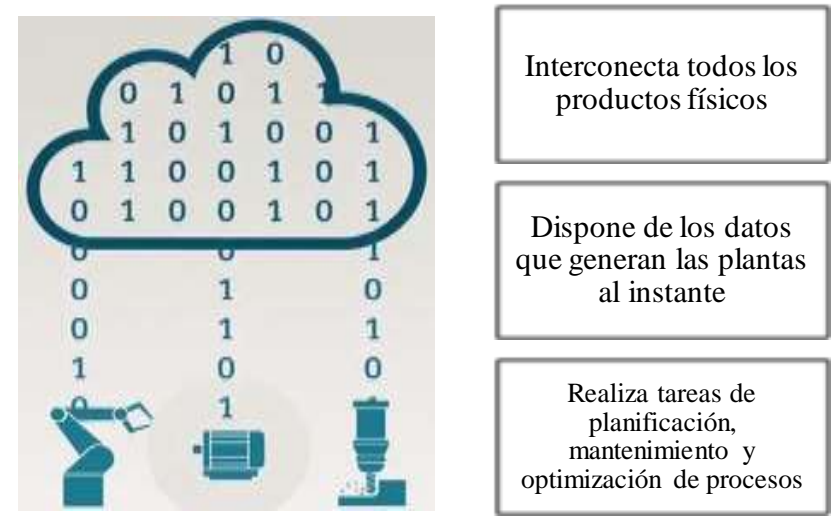

Dispone de los datos que generan las plantas

$$
\text { al instante }
$$

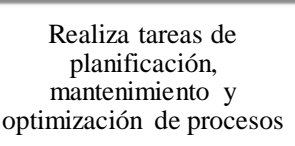

Figura 5 Adquisición de datos en la nube y Aplicaciones

\section{- IPC}

Computadoras industriales de alto rendimiento tienen capacidad hasta 1 TB de memoria RAM, Hasta 5 monitores, poner en red, en la figura 6 se muestra un mapa mental y sus aplicaciones.
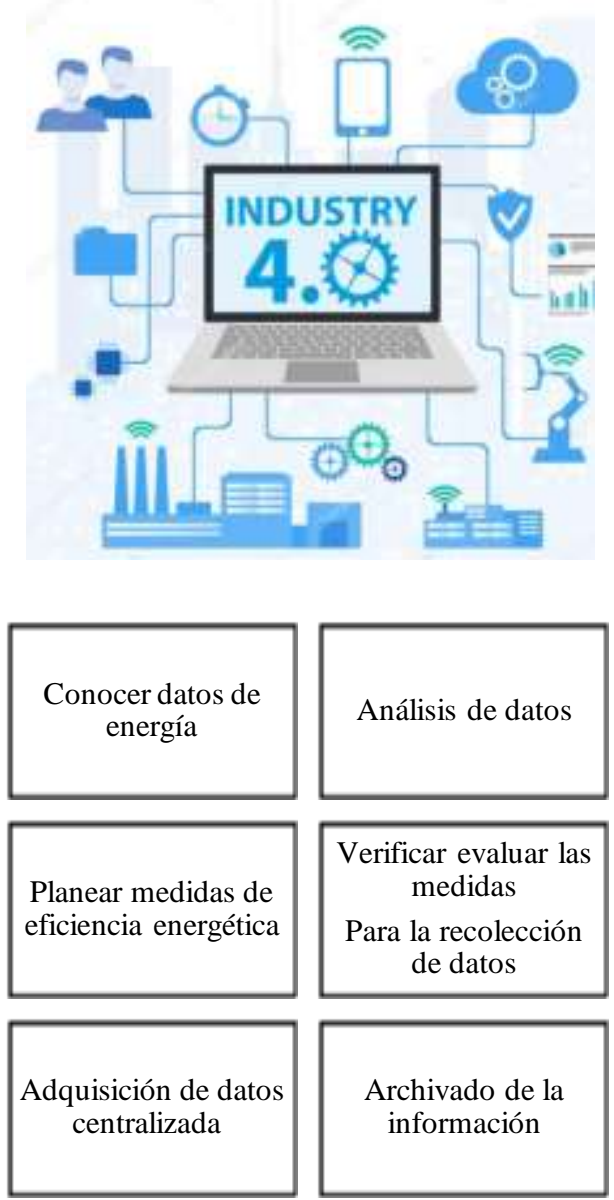

Archivado de la información

Figura 6 Computadoras Industriales y Aplicaciones

\section{- $\quad$ Sistemas SCADA}

El sistema SCADA es una herramienta de automatización y control industrial utilizada en los procesos productivos que puede controlar, supervisar, recopilar datos, analizar datos $\mathrm{y}$ generar informes a distancia mediante una aplicación informática. Su principal función es la de evaluar los datos con el propósito de subsanar posibles errores (Industría, 2018), la figura 7 muestra un sistema SCADA y sus aplicaciones.

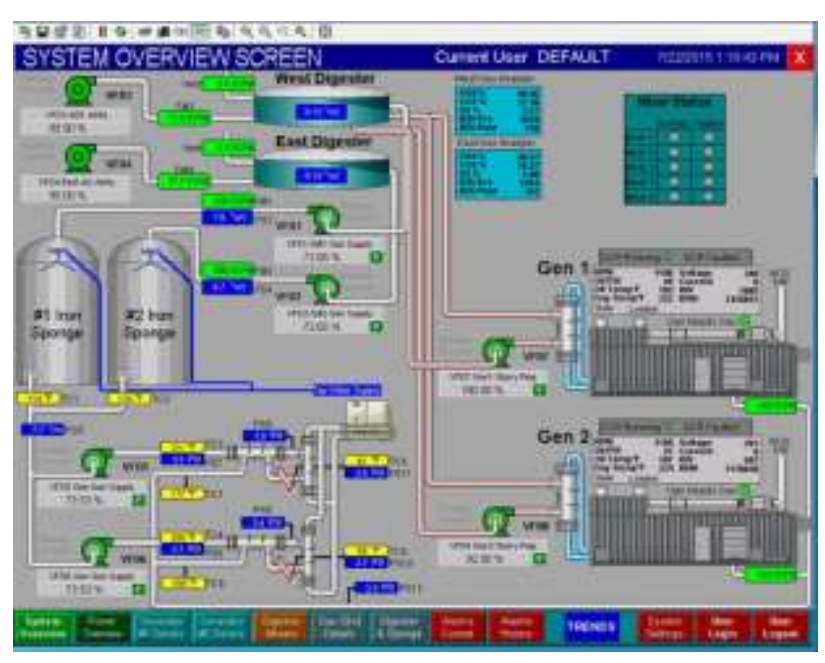




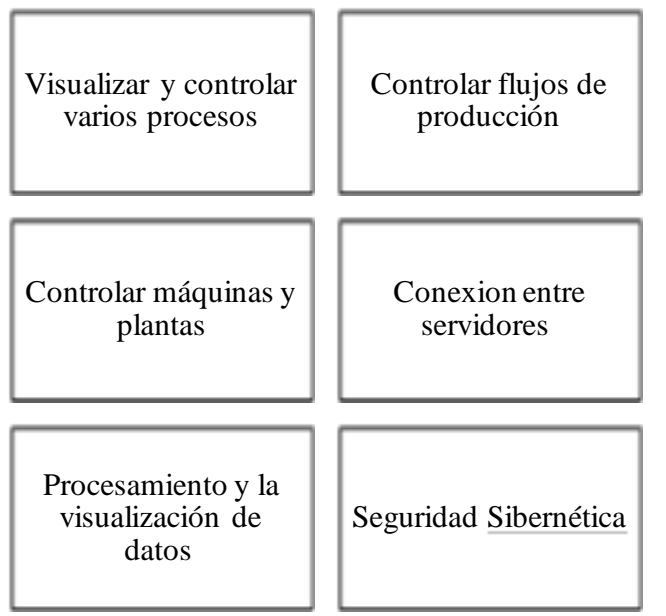

Figura 7 Panel View de un sistema SCADA y Aplicaciones

\section{Softwares de gestión de la energía}

A lo largo de la planta (Energy manager) el PLC apaga cargas que no son críticas en el proceso o pone en estados de reposo apagando la parte de potencia para bajo consumo (Siemens, PM-ANALIZAR, 2019), en la figura 8 se muestra el software y sus aplicaciones.
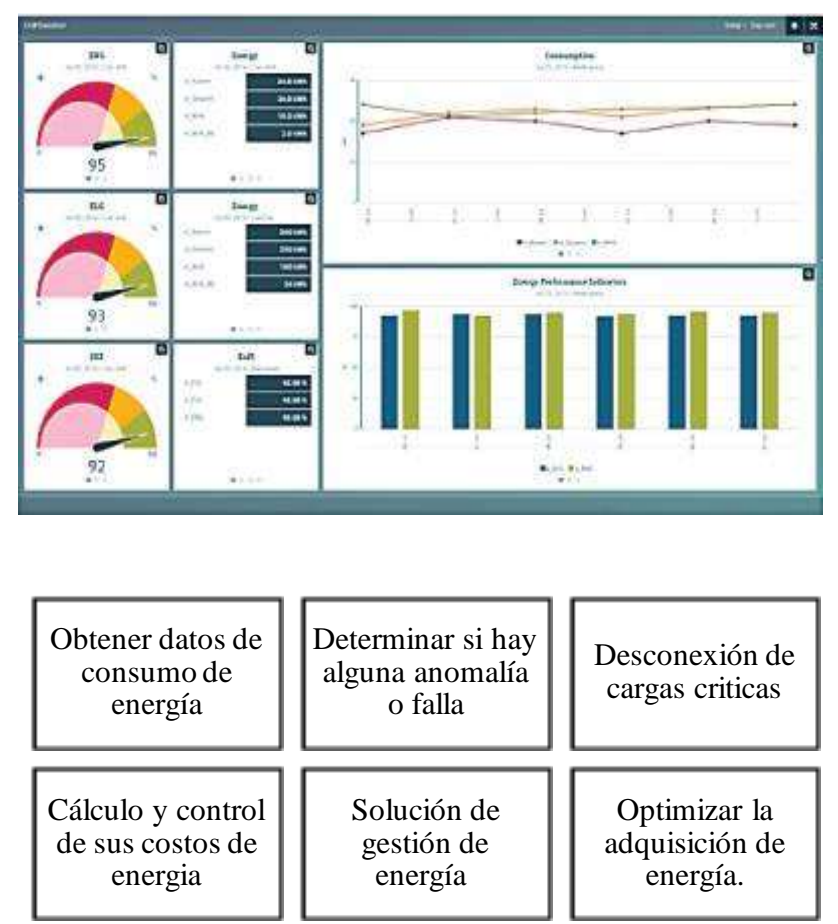

Figura 8 Software de consumo de energía Energy Manager y Aplicaciones

Fuente: Cortesía Siemens Win CC

\section{- $\quad$ Fabricación aditiva}

Con la impresión 3D, podemos fabricar nuestros propios componentes y mecanismos, en la figura 9 se muestra el sistema y sus aplicaciones.
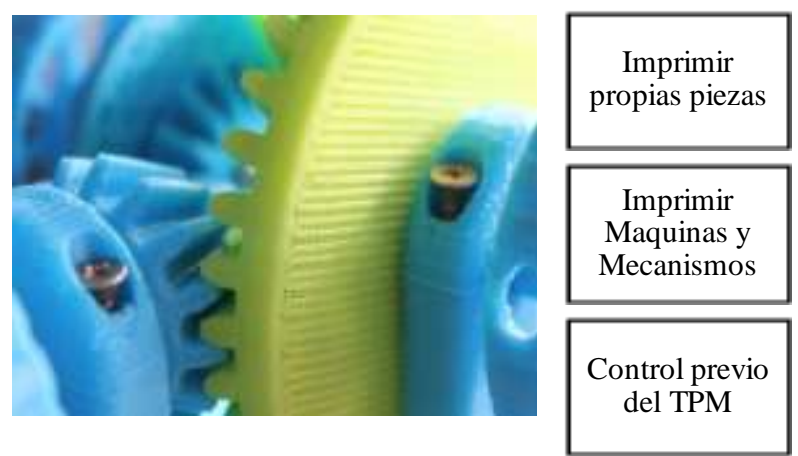

Figura 9 Engranes fabricados por impresión 3D y aplicaciones

\section{Robótica autónoma}

Los robots autónomos realizarán tareas de mantenimiento de forma autónoma, en la figura 10 se muestra un robot industrial y sus aplicaciones.
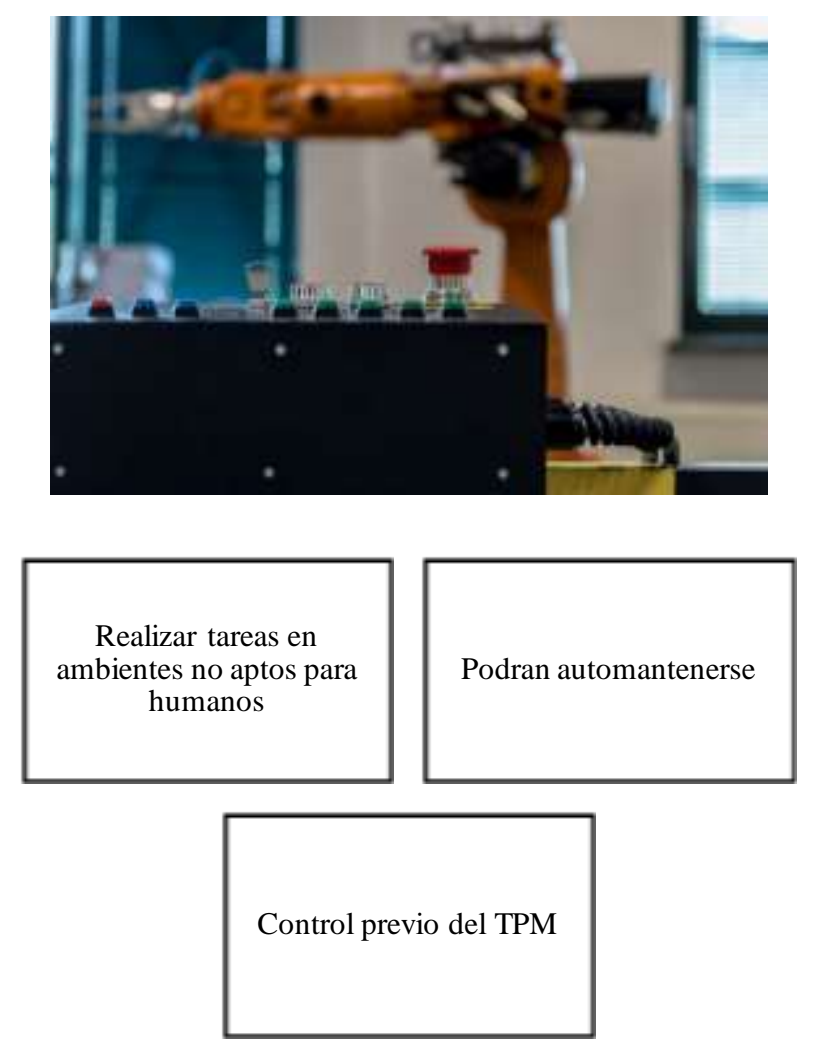

Figura 10 Robot Industrial y Aplicaciones

\section{La simulación y la realidad aumentada}

Las tareas de mantenimiento en las simulaciones de rendimiento, Simulan el comportamiento de las grasas, aerosoles y lubricantes, en base a factores externos, medioambientales, situaciones excepcionales, sucesos imprevistos o incluso fallos humanos. 


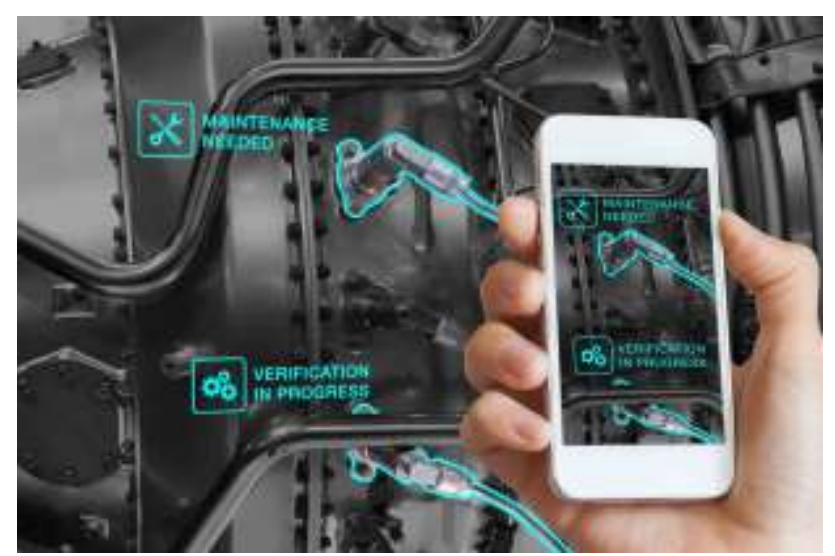

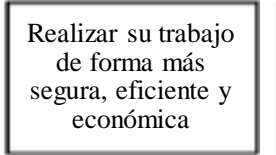

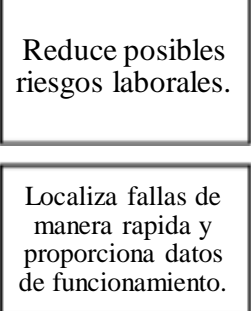

Desconexión de cargas criticas

Reduce los costes de mantenimiento.
Figura 11 Realidad Virtual en el mantenimiento y Aplicaciones

\section{Resultados}

Como resultado final se determina que nosotros como docentes de la carrera de Ingeniería en Mantenimiento Industrial aún no estamos preparados para formar profesionistas en cuanto a lo laboral, las competencias y habilidades requeridas por las empresas que emplean tecnologías de la industria 4.0, El mantenimiento Industrial maneja nuevas tecnologías como la fabricación aditiva, la conectividad, la robótica autónoma o incluso la simulación y la realidad aumentada, desde el punto de vista de la educación, la universidad debe de comenzar con un plan estratégico para preparar y formar personas con competencias relevantes hacia las temáticas que se desarrollan en el presente y hacia el futuro, pero primero dentro de este plan, debemos empezar con la capacitación de los docentes y de manera consecutiva con la adquisición de equipo de laboratorio acorde a la I4.0

\section{Desafíos Que se necesitan enfrentar para la industria 4.0:}

1. Conocimiento: Primero es necesario conocer a qué se refiere la industria 4.0, cómo se compone y cómo se utiliza, es decir, generar un plan de capacitación docente en Temas de la industria 4.0.
2. Preparación: Una vez que se cuenta con el conocimiento de la misma, es necesario capacitar a los docentes en tecnologías de la industria 4.0 para utilizarlas e integrarlas en la educación.

3. Aprendizaje: Los instructores de la institución deberán realizar estadías en las empresas para aprender $\mathrm{y}$ actualizarse sobre las nuevas tecnologías de la industria 4.0 para poder aplicarla en su campo.

4. Infraestructura: Finalmente es necesario actualizar los equipos de laboratorio de la institución con lo más nuevo en la industria 4.0, con la participación de los docentes expertos en el área para que realicen propuestas de equipamiento.

\section{Conclusiones}

El rol del docente es importante en la I4.0, porque son ellos los que formarán a las jóvenes para los empleos del futuro, en el cual necesitarán las competencias para resolver problemas más relevantes del mundo, pero, las instituciones educativas y los docentes no pueden hacerlo solos, es necesario la participación integral del sector productivo, gubernamental y educativo.

\section{Recomendaciones}

Primero, las Autoridades Universitarias deben de empezar con un plan estratégico para formar a nuestros docentes para que puedan transmitir todo el conocimiento necesario $y$ tengan la experiencia y las herramientas necesarias para hacerlo con enfoque I4.0, también es indispensable formar docentes competentes en habilidades blandas las cuales son: Creatividad para resolver problemas $\mathrm{y}$ retos complejos de manera colaborativa, inteligencia emocional, pensamiento crítico, innovación, emprendimiento y metodologías ágiles para crear nuevos modelos de negocio.

Segundo, también es necesario cambiar la forma y las metodologías con las que educamos. 
Superando la barrera generacional para utilizar herramientas tecnológicas con un nivel I4.0, los docentes deben de impulsarse a traer a las aulas la experiencia, la práctica, las herramientas y los retos para enseñar haciendo $\mathrm{y}$ construyendo, basados en las tendencias y tecnologías actuales de la I4.0.

Tercero, para lograr esta transformación se debe contar con el apoyo gubernamental, el cual debe entender el cambio y apropiarlo dentro de las instituciones educativas. Esto sería benéfico para afrontar algunos de los retos para que los planes y programas de estudio cumplan con el perfil de egreso que demanda el sector productivo.

\section{Agradecimiento}

En especial a la Universidad Tecnológica de Chihuahua por el apoyo en esta publicación.

\section{Referencias}

Garatu, G. (2016). Obtenido de ¿Qué es y qué aporta la Industria 4.0?: https://grupogaratu.com/que-es-y-que-aporta-laindustria-4-0/

Ibérica, A. (20 de 04 de 2018). ¿Qué importancia tiene el Mantenimiento Predictivo $4.0(\mathrm{PdM})$ para la fábrica inteligente? Obtenido de https://abaserp.com/es/news/mantenimiento-predictivo-40para-la-fabrica-inteligente

Siemens. (12 de 07 de 2019). Obtenido de El Sistema de Gestión de Mantenimiento inteligente:

https://w3.siemens.com/moms/human-machineinterface/en/visualization-

software/scada/wincc-addons/pm-

maint/pages/default.aspx

Siemens. (12 de 07 de 2019). Obtenido de Análisis de datos de Alarmas y Procesos.: https://w3.siemens.com/mcms/human-machineinterface/en/visualizationsoftware/scada/wincc-addons/pmanalyze/pages/default.aspx 